\title{
RCRA Toxicity Characterization of Discarded Electronic Devices
}

Stephen E. Musson ${ }^{\mathrm{a}, \mathrm{c}}$, Kevin N. Vann ${ }^{\mathrm{a}, \mathrm{d}}$, Yong Chul-Jang ${ }^{\mathrm{a}, \mathrm{b}, \mathrm{e}}$, Sarvesh Mutha ${ }^{\mathrm{a}, \mathrm{f}}$, Aaron Jordan $^{\mathrm{a}, \mathrm{g}}$, Brian Pearson ${ }^{\mathrm{a}, \mathrm{h}}$, and Timothy G. Townsend ${ }^{\mathrm{a}, *}$

\section{Total Pages: 28}

Figures: S1-S13

Tables: S1-S13 


\section{Supplemental Material}

The following supplemental material provides individual results for 13 electronics devices tested for the leaching of lead, iron, and zinc utilizing the toxicity characteristic leaching procedure or a modified form of the leaching procedure. The included figures present the composition, by weight, of the electronic device by major category. The tables present the leaching results. Methods listed are: $\mathrm{L}=$ large scale TCLP extraction, device simply disassembled, MS = modified TCLP extraction, device only disassembled, $\mathrm{SS}=$ standard regulatory TCLP extraction, device mechanically shredded then hand cut to meet size requirement, and True $=$ standard regulatory TCLP extraction, device hand cut to meet size requirement.

\section{TCLP Data}

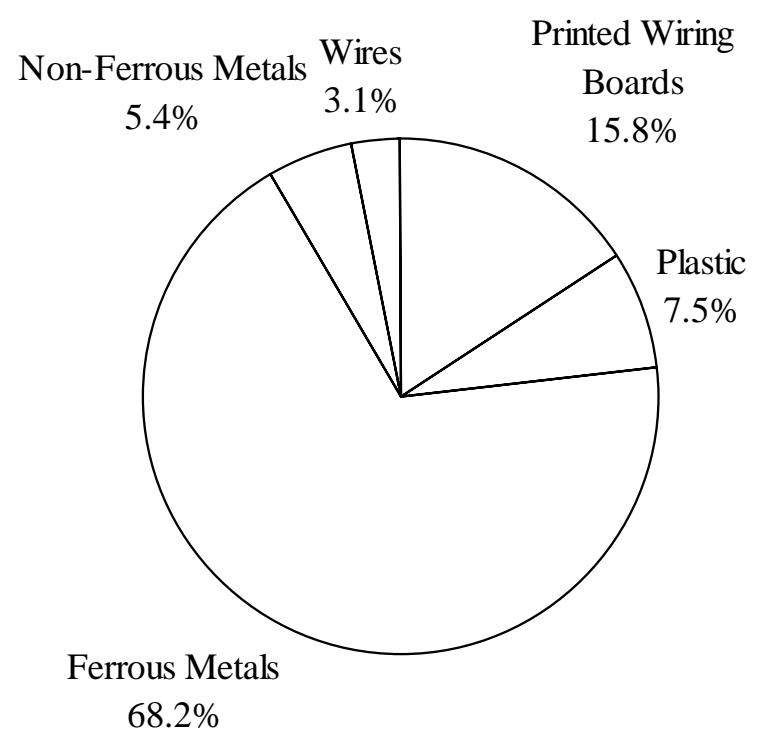

Figure S-0. Average CPU Composition of 29 Computer CPUs by Weight 
Table S-1. TCLP Results for CPUs

\begin{tabular}{|c|c|c|c|c|c|c|c|}
\hline \multirow{2}{*}{$\begin{array}{c}\text { Sampl } \\
\mathrm{e} \\
\text { Numbe }\end{array}$} & \multirow[t]{2}{*}{ Make } & Model/Year & \multirow[t]{3}{*}{ Method } & \multirow[t]{3}{*}{$\begin{array}{l}\mathrm{p} \\
\mathrm{H}\end{array}$} & \multicolumn{3}{|c|}{$\begin{array}{l}\text { Metal Concentration in } \\
\text { Leachate }(\mathrm{mg} / \mathrm{L})\end{array}$} \\
\hline & & & & & & & \\
\hline $\mathrm{r}$ & & & & & & & \\
\hline & & & & & $\mathrm{Fe}$ & $\mathrm{Pb}$ & $\mathrm{Zn}$ \\
\hline 1 & Sun Microsystems & SPARK Station 2/ 1993 & (S.S.) & 5. & 92.3 & 1.36 & 112 \\
\hline 2 & Sun Microsystems & SPARK Station 2/ 1993 & (S.S.) & 5. & 37.7 & 5.98 & 107 \\
\hline 3 & Sun Microsystems & SPARK Station 2/ 1993 & (S.S.) & 5. & 85.7 & 1.00 & 103 \\
\hline 4 & Sun Microsystems & SPARK Station 2/ 1993 & $(\mathrm{~L})$ & 5. & 104 & 8.55 & 143 \\
\hline 5 & Sun Microsystems & SPARK Station 2/ 1993 & (L) & 5. & 93.8 & 8.92 & 153 \\
\hline 6 & Sun Microsystems & SPARK Station 2/ 1993 & (L) & 5. & 92.6 & 8.26 & 156 \\
\hline 7 & Sun Microsystems & SPARK Station 2/ 1993 & (True) & 5. & 49.6 & 0.539 & 105 \\
\hline 8 & Sun Microsystems & SPARK Station 2/ 1993 & (True) & 5. & 11.4 & 0.367 & 118 \\
\hline 9 & Compaq & ProLinea 4/66 & (S.S.) & 5. & 106 & 1.13 & 84.0 \\
\hline 10 & Compaq & ProLinea 4/66 & (S.S.) & 5. & 85.2 & 0.855 & 122 \\
\hline 11 & Compaq & ProLinea 4/66/ 1995 & $(\mathrm{~L})$ & 5. & 255 & 5.46 & 128 \\
\hline 12 & Compaq & ProLinea 4/66/ 1994 & (True) & 5. & 17.5 & 0.309 & 147 \\
\hline 13 & IBM PS2 & 55SX/ 1987 & (S.S.) & 5. & 84.1 & 3.19 & 128 \\
\hline 14 & IBM PS2 & 55SX/ 1989 & $(\mathrm{~L})$ & 5. & 117 & 21.4 & 81.2 \\
\hline 15 & IBM PS2 & 55SX/ 1991 & (L) & 5. & 132 & 16.4 & 92.2 \\
\hline 16 & IBM PS2 & $55 \mathrm{SX}$ & (True) & 5. & 19.6 & 2.27 & 147 \\
\hline 17 & NCR & 6020 & (S.S.) & 5. & 119 & 0.965 & 99.4 \\
\hline 18 & NCR & $6020 / 1994$ & (L) & 5. & 127 & 9.53 & 103 \\
\hline 19 & NCR & $6020 / 1993$ & (True) & 5. & 23.7 & 0.431 & 130 \\
\hline 20 & NCR & 6020 & (True) & 5. & 31.2 & 0.527 & 122 \\
\hline 21 & Oli & M4 Module 464/ 1994 & (S.S.) & 5. & 96.2 & 3.59 & 42.9 \\
\hline 22 & Oli & M4 Module 464/ 1995 & (S.S.) & 5. & 136 & 1.48 & 52.4 \\
\hline 23 & Oli & M4 Module 464/ 1994 & $(\mathrm{~L})$ & 5. & 64.7 & 5.25 & 20.6 \\
\hline 24 & Oli & M4 Module 464/ 1995 & (L) & 5. & 24.3 & 3.07 & 33.5 \\
\hline 25 & Oli & M4 Module 464/ 1994 & (L) & 5. & 131 & 15.5 & 27.1 \\
\hline 26 & Oli & M4 Module 464/ 1994 & (L) & 5. & 62.5 & 4.04 & 34.3 \\
\hline 27 & Oli & M4 Module 464/ 1994 & (True) & 5. & 4.85 & 0.273 & 172 \\
\hline 28 & Oli & M4 Module 464/ 1994 & (True) & 5. & 58.7 & 3.06 & 116 \\
\hline 29 & Network General & Sniffer Server/ 1995 & (S.S.) & 5. & 111 & 1.28 & 85.7 \\
\hline 30 & Network General & Sniffer Server/ 1991 & $(\mathrm{~L})$ & 5. & 44.3 & 0.600 & 99.2 \\
\hline 31 & Network General & Sniffer Server/ 1996 & (L) & 5. & 49.7 & 0.489 & 101 \\
\hline 32 & Network General & Sniffer Server/ 1995 & (True) & 5. & 35.4 & 0.329 & 106 \\
\hline 33 & ATT Globalyst & $9011 / 1994$ & (S.S.) & 5. & 147 & 0.531 & 111 \\
\hline 34 & ATT Globalyst & $9011 / 1994$ & (L) & 5. & 189 & 9.12 & 114 \\
\hline 35 & ATT Globalyst & $9011 / 1994$ & (True) & 5. & 6.13 & 0.235 & 168 \\
\hline 36 & ATT Globalyst & $9011 / 1994$ & (True) & 5. & 19.3 & 0.135 & 129 \\
\hline 37 & Compaq & Prolinea 4/33 1994 & $(\mathrm{~L})$ & 5. & 201 & 8.41 & 215 \\
\hline
\end{tabular}




$\begin{array}{lccccccc}38 & \text { Compaq } & \text { Prolinea 4/33 1993 } & \text { (L) } & 5 . & 253 & 7.10 & 160 \\ 39 & \text { Compaq } & \text { Prolinea 4/33 1994 } & \text { (L) } & 5 . & 267 & 6.59 & 134 \\ 40 & \text { Compaq } & \text { Prolinea 4/33 1994 } & \text { (True) } & 5 . & 44.2 & 0.476 & 220 \\ 41 & \text { Fortiva 5000 } & \text { CPC-2803/1994 } & \text { (L) } & 5 . & 5.74 & 0.687 & 95.1 \\ 42 & \text { Digital DEC pc } & 806 W W / 1994 & \text { (L) } & 5 . & 2.90 & 2.79 & 82.0 \\ 43 & \text { IBM } & 55 S X / 1989 & \text { (L) } & 5 . & 131 & 12.6 & 69.7 \\ 44 & \text { IBM } & 55 S X / 1990 & \text { (L) } & 5 . & 155 & 13.4 & 101 \\ 45 & \text { NCR } & 1993 & \text { (L) } & 5 . & 150 & 6.49 & 120 \\ 46 & \text { e Machines } & \text { 366i2/1999 } & \text { (L) } & 5 . & 13.6 & 0.241 & 181 \\ 47 & \text { Gateway } & \text { 4DX33/1993 } & \text { (L) } & 5 . & 24.9 & 0.202 & 127 \\ 48 & \text { Dimension XPS } & \text { MM8/1996 } & \text { (L) } & 5 . & 68.0 & 4.99 & 75.9 \\ 49 & \text { Dimension XPS } & \text { MM8/1997 } & \text { (L) } & 5 . & 4.93 & 0.353 & 85.3 \\ 50 & \text { Dimension XPS } & \text { MM8/1996 } & \text { (L) } & 5 . & 4.63 & 0.281 & 82.2 \\ 51 & \text { Dimension XPS } & \text { MM8/1997 } & \text { (L) } & 5 . & 8.83 & 0.331 & 87.4 \\ 52 & \text { Compaq } & \text { PS-5151-4A/1996 } & \text { (L) } & 5 . & 11.0 & 0.242 & 88.5 \\ 53 & \text { Compaq } & \text { PS-5151-4A/1996 } & \text { (L) } & 5 . & 34.7 & 0.184 & 87.4 \\ 54 & \text { Dell } & 450-L / 1993 & \text { (L) } & 5 . & 135 & 5.00 & 106 \\ 55 & \text { Gateway } & 4 D \text { X2-66/1994 } & \text { (L) } & 5 . & 62.3 & 1.83 & 113 \\ 56 & \text { Apple } & \text { M3098/1995 } & \text { (L) } & 5 . & 122 & 10.1 & 55.1 \\ 57 & \text { Dell } & \text { DCS/1998 } & \text { (L) } & 5 . & 10.8 & 0.237 & 191 \\ 58 & \text { NEC } & 1996 & \text { (L) } & 5 . & 201 & 8.95 & 133 \\ 59 & \text { Dell } & \text { Optiplex GL 5100/1996 } & \text { (L) } & 5 . & 73.0 & 1.87 & 98.4 \\ 60 & \text { Dell } & \text { Optiplex GL 5100/1996 } & \text { (L) } & 5 . & 121 & 11.2 & 114 \\ 61 & \text { Dell } & \text { Optiplex GN/1998 } & \text { (L) } & 5 . & 13.0 & 0.288 & 204 \\ 62 & \text { Gateway } & \text { E 3000/1997 } & \text { (L) } & 5 . & 49.5 & 1.66 & 117 \\ 63 & \text { Dell } & \text { Optiplex GN/1998 } & \text { (L) } & 5 . & 8.11 & 0.278 & 202 \\ 64 & \text { Dell } & \text { Optiplex GS/1997 } & \text { (L) } & 5 . & 8.23 & 0.398 & 199\end{array}$




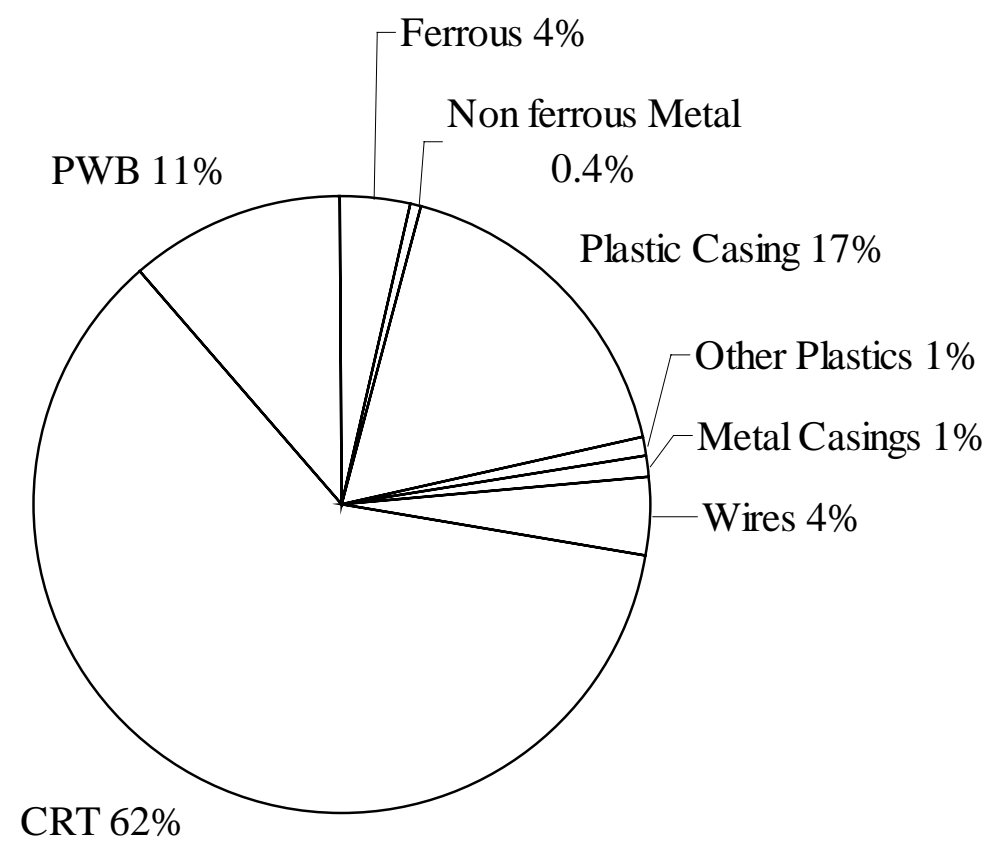

Figure S-2. Average Composition of Computer Monitors Tested 
Table S-2. TCLP Results for Computer Monitors

\begin{tabular}{|c|c|c|c|c|c|c|c|}
\hline \multirow[t]{2}{*}{$\begin{array}{l}\text { Sample } \\
\text { Number }\end{array}$} & \multirow[t]{2}{*}{ Make } & \multirow[t]{2}{*}{ Model/Year } & \multirow[t]{2}{*}{ Method } & \multirow[t]{2}{*}{$\mathrm{PH}$} & \multicolumn{3}{|c|}{$\begin{array}{c}\text { Metal Concentration in } \\
\text { Leachate } \\
(\mathrm{mg} / \mathrm{L})\end{array}$} \\
\hline & & & & & $\mathrm{Fe}$ & $\mathrm{Pb}$ & $\mathrm{Zn}$ \\
\hline 1 & Dell & Ultrascan15FS & (L) & 5.1 & 107 & 12.8 & 9.09 \\
\hline 2 & Gateway & CS1572DG & (L) & 5.1 & 127 & 32.9 & 21.3 \\
\hline 3 & Packard & 2025 & (L) & 5.1 & 62.5 & 59.0 & 12.5 \\
\hline 4 & Gateway & 500-069CS & (L) & 5.1 & 122 & 40.2 & 25.9 \\
\hline 5 & Compaq & 471 & (L) & 5.0 & 105 & 13.0 & 30.8 \\
\hline 6 & Samsung & CJ4681 & (L) & 5.0 & 120 & 33.1 & 27.9 \\
\hline 7 & Phillips & NR & (L) & 5.0 & 93.7 & 93.7 & 19.8 \\
\hline 8 & Gateway & 500-069CS & (L) & 5.1 & 108 & 50.2 & 23.2 \\
\hline 9 & Apple & NR & (L) & 5.1 & 125 & 95.0 & 15.5 \\
\hline 10 & Dell & E551 & (L) & 5.0 & 167 & 59.2 & 33.7 \\
\hline
\end{tabular}




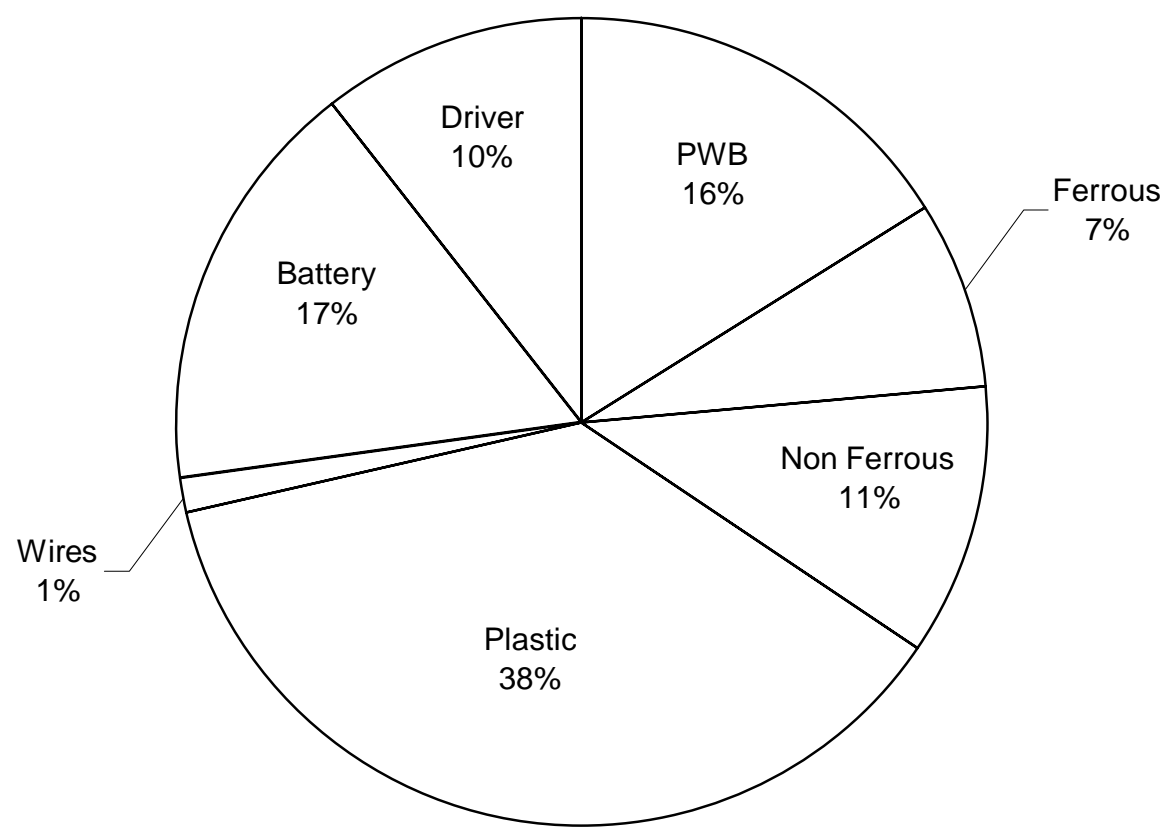

Figure S-3. Average Composition of Laptops Tested 
Table S-3. TCLP Results for Laptops

\begin{tabular}{|c|c|c|c|c|c|c|c|c|}
\hline \multirow[t]{2}{*}{$\begin{array}{c}\text { Sample } \\
\text { Number }\end{array}$} & \multirow[t]{2}{*}{ Make } & \multirow[t]{2}{*}{ Model/Year } & \multirow[t]{2}{*}{ Method } & \multirow[t]{2}{*}{ pH } & \multicolumn{4}{|c|}{$\begin{array}{l}\text { Metal Concentration in } \\
\text { Leachate }(\mathrm{mg} / \mathrm{L})\end{array}$} \\
\hline & & & & & $\mathbf{H g}$ & $\mathbf{F e}$ & $\mathbf{P b}$ & $\mathbf{Z n}$ \\
\hline 1 & Compaq & Compaq LTE 5000 (series & (L) & 5.2 & $<0.03$ & 53.6 & 17.2 & 47.0 \\
\hline 2 & IBM & IBM Thinkpad 9545/1995 & (L) & 5.1 & $<0.03$ & 66.8 & 25.6 & 16.0 \\
\hline 3 & NEC & NEC Versa $\mathrm{m} / 100$ & (L) & 5.0 & $<0.03$ & 100 & 34.5 & 20.8 \\
\hline 4 & Compaq & Compaq LTE Lite $4 / 25 \mathrm{E}$ & (L) & 5.0 & $<0.03$ & 82.0 & 25.7 & 15.0 \\
\hline 5 & Compaq & Compaq LTE 5280 (Series & (L) & 5.0 & $<0.03$ & 80.7 & 21.2 & 42.0 \\
\hline 6 & Zenith & Zenith ZWL-184-97 & (L) & 5.0 & $<0.03$ & 17.3 & 18.4 & 53.2 \\
\hline 7 & Toshiba & Toshiba T1200 System Unit & (L) & 5.1 & $<0.03$ & 80.2 & 14.6 & 54.8 \\
\hline 8 & Zenith & Zenith ZWL-371-A & (L) & 5.1 & $<0.03$ & 182 & 25.0 & 28.0 \\
\hline 9 & Zenith & Zenith ZWL-371-A & (L) & 5.0 & $<0.03$ & 206 & 27.9 & 38.0 \\
\hline 10 & IBM & Thinkpad 9545 & (True) & 5.0 & $<0.03$ & 0.800 & 36.5 & 0.500 \\
\hline 11 & Compaq & Contura Series, 4/25C (2820) & (True) & 5.2 & $<0.03$ & 72.0 & 41.5 & 11.0 \\
\hline 12 & NEC & VERSA m/100 (PC-580- & (True) & 5.0 & $<0.03$ & 46.0 & 86.2 & 1.00 \\
\hline 13 & IBM & Thinkpad 350c & (True) & 5.0 & $<0.03$ & 116 & 11.4 & 51.0 \\
\hline 14 & Dell & $325 \mathrm{~N}$ & (True) & 5.0 & $<0.03$ & 91.0 & 26.9 & 42.0 \\
\hline 15 & AST & Premium Exec 386 SX/20 & (True) & 5.0 & $<0.03$ & 39.0 & 19.3 & 152 \\
\hline
\end{tabular}




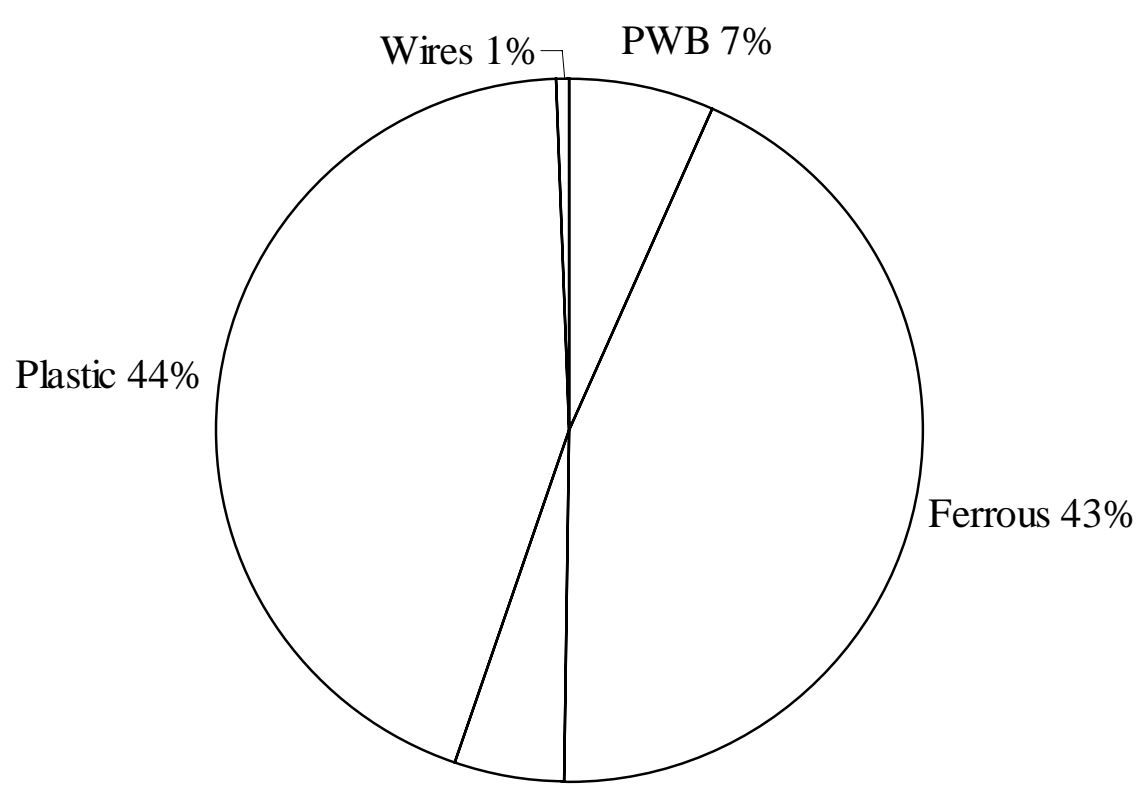

Nonferrous 5\%

Figure S-4. Average Composition of Printers Tested 


\begin{tabular}{|c|c|c|c|c|c|c|c|}
\hline \multirow{3}{*}{$\begin{array}{l}\text { Sample } \\
\text { Number }\end{array}$} & \multicolumn{7}{|c|}{ Table S-4. TCLP Results for Printers } \\
\hline & Make & Model & Method & $\mathbf{p H}$ & \multicolumn{3}{|c|}{$\begin{array}{l}\text { Metal Concentration in } \\
\text { Leachate }(\mathrm{mg} / \mathrm{L})\end{array}$} \\
\hline & & & & & $\mathbf{F e}$ & $\mathbf{P b}$ & $\mathbf{Z n}$ \\
\hline 1 & Okidata & EN2700A & (L) & 5.0 & 114 & 6.00 & 49.3 \\
\hline 2 & HP Deskjet & $\mathrm{C} 4567 \mathrm{~A}$ & (L) & 5.1 & 47.8 & 0.800 & 97.8 \\
\hline 3 & HP office jet & C3801 & (L) & 5.1 & 231 & 6.61 & 54.0 \\
\hline 4 & HP Deskjet 890C & $\mathrm{C} 5876 \mathrm{~A}$ & (L) & 5.0 & 176 & 3.60 & 43.4 \\
\hline 5 & HP Desk writer & 2279A & (L) & 5.0 & 101 & 3.63 & 53.7 \\
\hline 6 & HP Deskjet 550C & $\mathrm{C} 2121 \mathrm{~A}$ & (L) & 5.1 & 196 & 5.85 & 34.8 \\
\hline 7 & HP Deskjet 500C & C2106A & (L) & 5.1 & 131 & 6.64 & 60.6 \\
\hline 8 & HP Deskjet 672C & C2164A & (L) & 5.0 & 7.48 & 0.316 & 46.0 \\
\hline 9 & Panasonic Quiet & N/R & (L) & 5.1 & 174 & 5.45 & 63.9 \\
\hline 10 & HP Deskjet 832C & C6413B & (L) & 5.2 & 316 & 1.91 & 106 \\
\hline
\end{tabular}




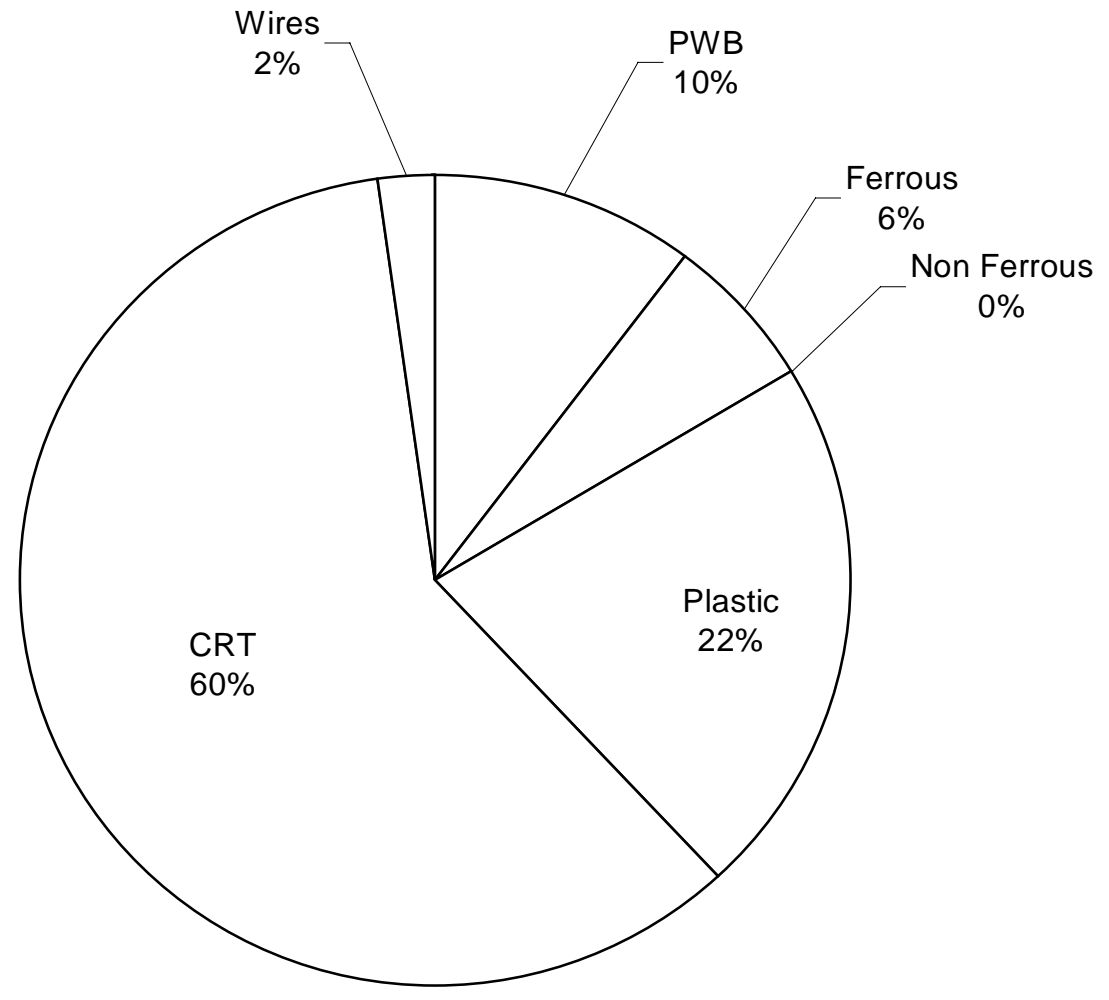

Figure S-5. Average Composition of Color TVs Tested 
Table S-5. TCLP Results for Color TV

\begin{tabular}{|c|c|c|c|c|c|c|c|}
\hline \multirow{2}{*}{$\begin{array}{c}\text { Sample } \\
\text { Number }\end{array}$} & \multirow[t]{2}{*}{ Make } & \multirow[t]{2}{*}{ Model/Year } & \multirow[t]{2}{*}{ Method } & \multirow[t]{2}{*}{ pH } & \multicolumn{3}{|c|}{$\begin{array}{l}\text { Metal Concentration in } \\
\text { Leachate }(\mathrm{mg} / \mathrm{L})\end{array}$} \\
\hline & & & & & $\mathbf{F e}$ & $\mathbf{P b}$ & $\mathbf{Z n}$ \\
\hline 1 & JVC & C-13WL5/1994 & (L) & 5.1 & 68.0 & 31.0 & 23.0 \\
\hline 2 & Panasonic & СТ 216-A/1987 & (L) & 5.3 & 39.8 & 15.0 & 53.7 \\
\hline 3 & Philips & RAX 150WA02/1990 & (L) & 5.2 & 95.5 & 15.4 & 21.2 \\
\hline 4 & Sanyo & 1999 & (L) & 5.1 & 124 & 12.0 & 13.4 \\
\hline 5 & RCA & 2001 & (L) & 5.0 & 140 & 43.5 & 17.6 \\
\hline 6 & Zenith & D1312W, 1987 & (L) & 5.1 & 135 & 28.6 & 15.0 \\
\hline
\end{tabular}




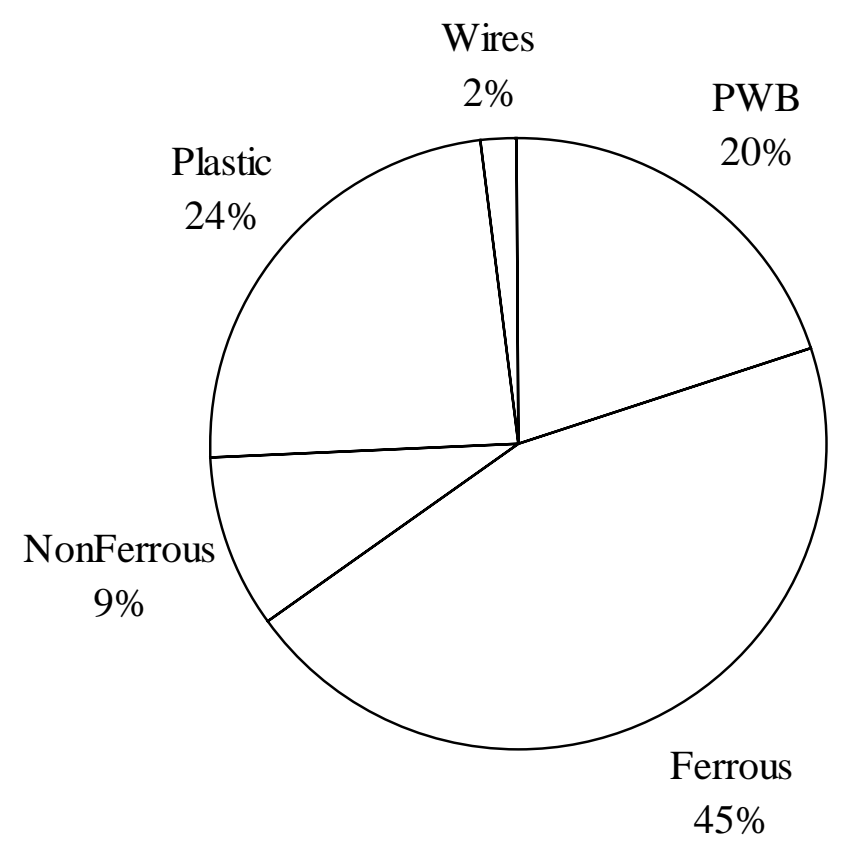

Figure S-6. Average Composition of VCRs Tested 
Sample
Number

1

2

3

4

5

6

7

8

9

10
Make

Sylvania

Philips

Memorex

JVC

JC Penny

Emerson/Hitachi

Phillips

Hitachi/JVC

Sharp

Phillips
Table S-6. TCLP Results of VCRs

Model

Method pH

H1600VD
VRA631AT22
MVR4040
HRDX420
686-6054
VCR755/VT-M262A
VR6615AT01
HR-J600U
XA-905
VRX262AT22

Metal Concentration in Leachate $(\mathrm{mg} / \mathrm{L})$ $\begin{array}{lll}\mathrm{Fe} & \mathrm{Pb} & \mathrm{Zn}\end{array}$

$\begin{array}{lllll}\text { (L) } & 5.1 & 150 & 10.9 & 34.9\end{array}$

$\begin{array}{lllll}\text { (L) } & 5.5 & 56.3 & 8.40 & 51.9\end{array}$

$\begin{array}{llll}\text { (L) } \quad 5.2 & 230 & 9.91 & 58.1\end{array}$

$\begin{array}{lllll}\text { (L) } \quad 5.4 & 236 & 12.6 & 152\end{array}$

$\begin{array}{lllll}\text { (L) } & 5.0 & 105 & 8.27 & 46.9\end{array}$

$\begin{array}{lllll}\text { (L) } & 5.0 & 10.4 & 1.39 & 92.4\end{array}$

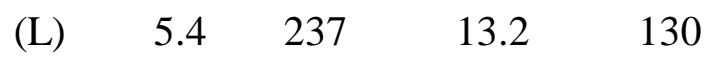

$\begin{array}{lllll}\text { (L) } & 5.2 & 107 & 11.3 & 147\end{array}$

$\begin{array}{lllll}\text { (L) } & 5.2 & 237 & 19.3 & 164\end{array}$

$\begin{array}{lllll}\text { (L) } & 5.2 & 161 & 17.7 & 65.5\end{array}$ 


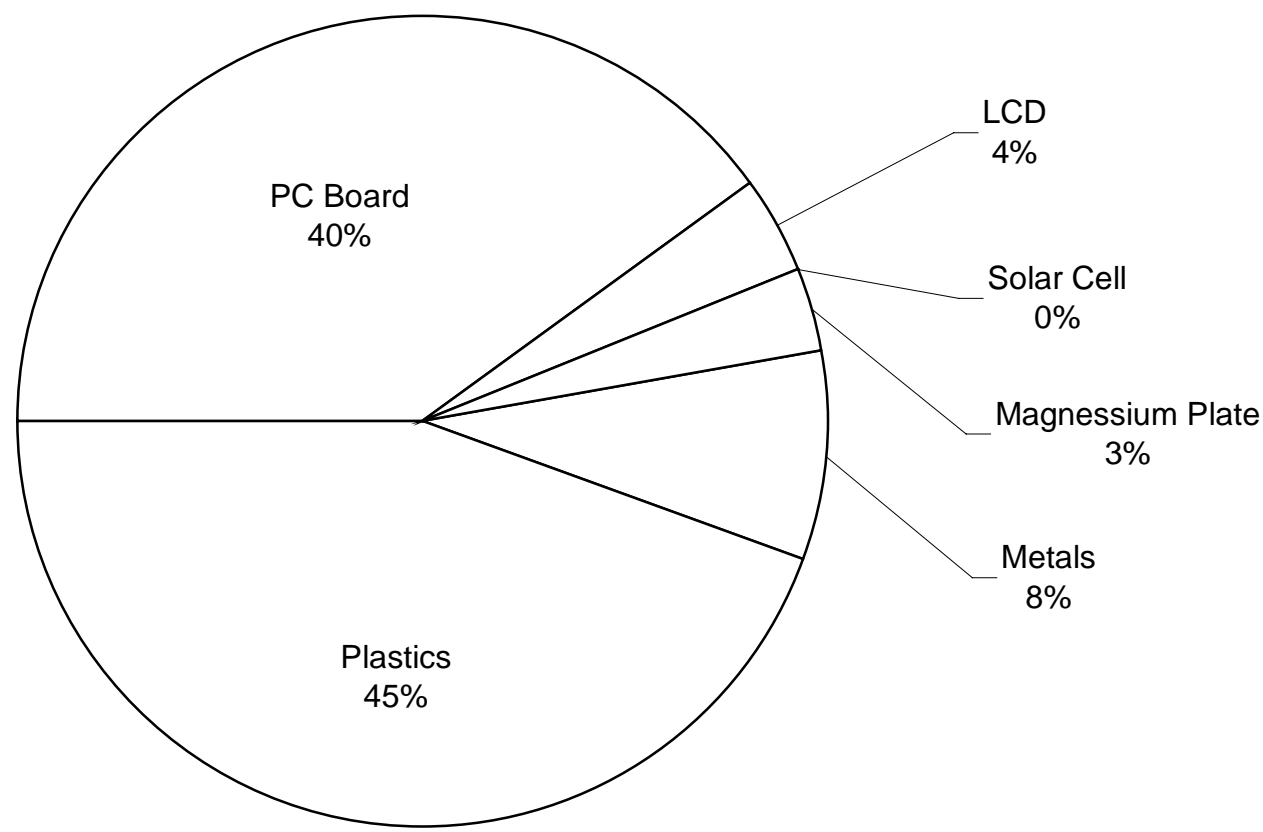

Figure S-7. Average Composition of Cell Phones Tested 
Table S-7. TCLP Results for Cell Phones

\begin{tabular}{|c|c|c|c|c|c|c|c|}
\hline \multirow[t]{2}{*}{$\begin{array}{l}\text { Sample } \\
\text { Number }\end{array}$} & \multirow[t]{2}{*}{ Make } & \multirow[t]{2}{*}{ Model/Year } & \multirow[t]{2}{*}{ Method } & \multirow[t]{2}{*}{ pH } & \multicolumn{3}{|c|}{$\begin{array}{l}\text { Metal Concentration in } \\
\text { Leachate }(\mathrm{mg} / \mathrm{L})\end{array}$} \\
\hline & & & & & $\mathbf{F e}$ & $\mathbf{P b}$ & Zn \\
\hline 1 & Motorola & SWF4018DF J 160017B & (M.S.) & 4.9 & 43.4 & 141 & 6.34 \\
\hline 2 & Motorola & 80148WNBEA & (M.S) & 5.0 & 86.6 & 115 & 0.427 \\
\hline 3 & Sprint & QCP-2700 & (M.S) & 5.0 & 5.36 & 1.52 & 17.3 \\
\hline 4 & Motorola & SWF2049A H7 41843A4C & (M.S) & 4.9 & 5.89 & 1.64 & 3.14 \\
\hline 6 & Ericsson & CF768 & (M.S) & 5.0 & 43.6 & 115 & 18.9 \\
\hline 7 & Nokia & 2120 & (M.S) & 6.3 & 2.12 & 0.130 & 12.1 \\
\hline 8 & Nokia (b) & 5160 & (M.S) & 4.9 & 9.12 & 10.6 & 20.3 \\
\hline 9 & Nokia $(p)$ & 5160 & (M.S) & 5.0 & 2.33 & 1.87 & 18.3 \\
\hline 10 & Motorola & Piper 34106NNDPA/1996 & (M.S) & 4.9 & 107 & 13.9 & 4.45 \\
\hline 11 & Motorola & Piper 34106NNDPA/1996 & (M.S) & 4.9 & 92.6 & 15.1 & 3.90 \\
\hline 12 & Motorola & Piper 34106NNDPA/1996 & (M.S) & 4.9 & 119 & 17.1 & 4.14 \\
\hline 13 & Motorola & Piper 34106NNDPA/1996 & (M.S) & 4.9 & 113 & 16.7 & 2.72 \\
\hline 14 & Motorola & Piper 34106NNDPA/1996 & (M.S) & 4.9 & 125 & 15.2 & 3.13 \\
\hline 15 & Motorola & Piper 34106NNDPA/1996 & (M.S) & 4.9 & 111 & 15.5 & 0.300 \\
\hline 16 & Motorola & Piper 34106NNDPA/1996 & (True) & 4.9 & 109 & 19.8 & 3.45 \\
\hline 17 & Motorola & Piper 34106NNDPA/1996 & (True) & 4.9 & 109 & 21.4 & 4.14 \\
\hline 18 & Motorola & Piper 34106NNDPA/1996 & (True) & 4.9 & 97.3 & 16.1 & 3.09 \\
\hline 19 & Motorola & Piper 34106NNDPA/1996 & (True) & 4.9 & 93.9 & 17.2 & 3.12 \\
\hline 20 & Motorola & Piper 34106NNDPA/1996 & (True) & 4.9 & 105 & 19.8 & 3.27 \\
\hline 21 & Motorola & Piper 34106NNDPA/1996 & (True) & 4.9 & 83.2 & 17.9 & 2.50 \\
\hline 22 & Ericsson & DF688/1997 & (True) & 5.5 & 6.61 & 4.23 & 3.89 \\
\hline 23 & NEC & MP5G1A1A-1A/1996 & (True) & 5.4 & 3.52 & 2.90 & 7.04 \\
\hline 24 & Nokia & $2160 \mathrm{EFR}$ & (True) & 8.8 & 0.0978 & 0.0107 & 0.246 \\
\hline 25 & Ericsson & KH668(2)/1997 & (True) & 5.6 & 14.5 & 15.6 & 7.68 \\
\hline 26 & Nokia & 3390 & (True) & 5.0 & 55.5 & 59.8 & 18.9 \\
\hline 27 & NEC & DM2100 MP5G1B1-1A & (True) & 8.4 & 0.118 & 0.0048 & 0.047 \\
\hline 28 & Nokia & $5120 \mathrm{i}$ & (True) & 5.0 & 56.5 & 16.3 & 16.0 \\
\hline 29 & Motorola & Piper 76362NNDBA/1995 & (True) & 5.1 & 121 & 30.5 & 3.07 \\
\hline 30 & Mitsubish & G100e/1996 & (True) & 5.1 & 20.3 & 21.4 & 15.4 \\
\hline 31 & Qualcom & QCP-820(2) & (True) & 5.2 & 147 & 12.3 & 25.1 \\
\hline 32 & Ericsson & KH688/1997 & (True) & 5.4 & 17.3 & 1.08 & 7.26 \\
\hline
\end{tabular}




\begin{tabular}{|c|c|c|c|c|c|c|c|}
\hline 33 & Qualcom & QCP-820(1) & (True) & 5.0 & 0.599 & 1.53 & 20.5 \\
\hline 34 & Nokia & $6160 / 1997$ & (True) & 5.0 & 0.273 & 32.2 & 12.8 \\
\hline 35 & Nokia & $2180 / 1995$ & (True) & 9.0 & 0.0021 & 0.0326 & 0.145 \\
\hline 36 & $\mathrm{NEC}$ & TR5E800-26B Portable & (True) & 5.0 & 0.101 & 64.7 & 2.37 \\
\hline 37 & Motorola & FR50/2001 & (True) & 5.0 & 0.294 & 5.53 & 85.0 \\
\hline 38 & Nokia & $918+$ & (True) & 8.4 & 0.0013 & $<0.001$ & 0.053 \\
\hline 39 & LGIC & LGP-2300W & (True) & 5.0 & 0.226 & 22.9 & $5 . \hat{4} 1$ \\
\hline 40 & Nokia & $6160 \mathrm{i}$ & (True) & 5.0 & 0.299 & 31.3 & 13.4 \\
\hline 41 & Nokia & $6160(2) / 1997$ & (True) & 5.0 & 0.420 & 15.8 & 19.6 \\
\hline 42 & NEC & MP5AF4-1A Portable/1995 & (True) & 5.0 & 0.0936 & 61.4 & 15.1 \\
\hline 43 & Motorola & $\begin{array}{c}\text { Micro Elite TAC Lite II } \\
\text { 76096WHAPA/96 }\end{array}$ & (True) & 5.0 & 21.4 & 21.4 & 8.32 \\
\hline 44 & Motorola & $\begin{array}{c}\text { Ultra Classic } \\
\text { F09NFD8402AG/1990 }\end{array}$ & (True) & 5.0 & 0.0647 & 24.2 & 1.56 \\
\hline 45 & Motorola & $\begin{array}{c}\text { Profile } 300 \\
\text { 34906NNKBA/1997 }\end{array}$ & (True) & 5.0 & 69.0 & 35.0 & 0.688 \\
\hline 46 & Motorola & 76254NNFDA/1991 & (True) & 4.9 & 6.30 & 46.8 & 1.54 \\
\hline 47 & Motorola & Micro TAC Elite(2) & (True) & 5.0 & 58.6 & 13.3 & 6.83 \\
\hline 48 & Motorola & $\begin{array}{l}\text { 76732NNXEA/1995 } \\
\text { Micro TAC Elite } \\
\text { 76732NNEXA/1995 }\end{array}$ & (True) & 5.0 & 77.7 & 17.5 & 5.84 \\
\hline 49 & Qualcom & QCP-820(3) & (True) & 5.0 & 74.3 & 1.37 & 10.7 \\
\hline 50 & Motorola & F09HLD8415BG/1991 & (True) & 5.0 & 23.3 & 48.2 & 15.7 \\
\hline 51 & Motorola & F09HGD8467CG/1989 & (True) & 5.0 & 20.5 & 39.6 & 1.47 \\
\hline 52 & Motorola & 34106NNRSA/1995 & (True) & 5.0 & 65.8 & 19.3 & 5.98 \\
\hline 53 & Nokia & 2160EFR(2)/1994 & (True) & 9.4 & 1.70 & 0.315 & 7.81 \\
\hline
\end{tabular}




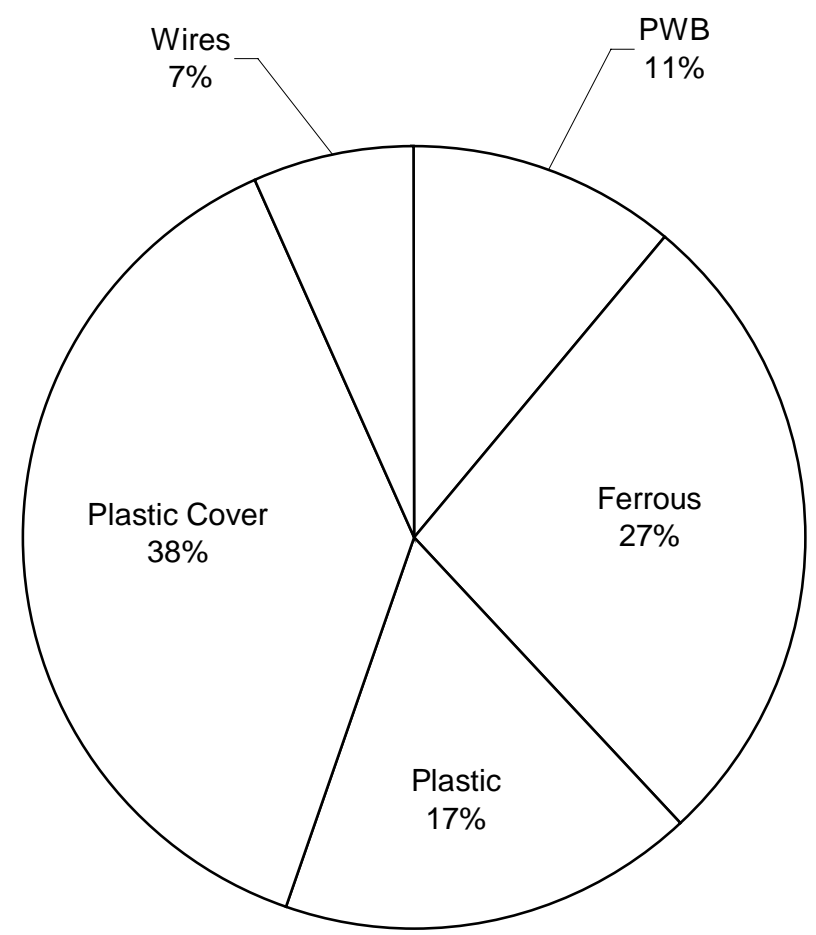

Figure S-8. Average Composition of Keyboards Tested 


\begin{tabular}{cccccccc}
$\begin{array}{c}\text { Sample } \\
\text { Number }\end{array}$ & Make & Model & Method & pH & \multicolumn{3}{c}{$\begin{array}{c}\text { Metal Concentration in } \\
\text { Leachate } \\
\text { (mg/L) }\end{array}$} \\
& & & & & & \multicolumn{3}{c}{ Pb } & Zn \\
& & & & & & Fe & Pbults for Keyboards \\
1 & NMB & RT101+ & (L) & 5.1 & 78.0 & 20.4 & 144 \\
2 & NMB & RT101+ & True & 5.1 & 16.0 & 2.28 & 164 \\
3 & NMB & RT101+ & True & 5.1 & 29.0 & 4.71 & 140 \\
4 & Gateway & SK-9921 & True & 5.1 & 116 & 0.100 & 73.6 \\
5 & Fujitsu & FKB 4700 & True & 5.1 & 68.0 & 0.110 & 77.2 \\
6 & Dell & SK1000REW & (L) & 5.1 & 119 & 0.610 & 40.4 \\
7 & Dell & RT7D5JTW & True & 5.1 & 59.8 & 0.620 & 52.5 \\
8 & Fujitsu & FKB 4700 & (L) & 5.1 & 105 & 1.63 & 50.8 \\
9 & Dell & RT7D5JTW & (L) & 5.1 & 276 & 1.00 & 74.3 \\
10 & IBM & 42H1292 & (L) & 5.1 & 155 & 14.1 & 51.5
\end{tabular}




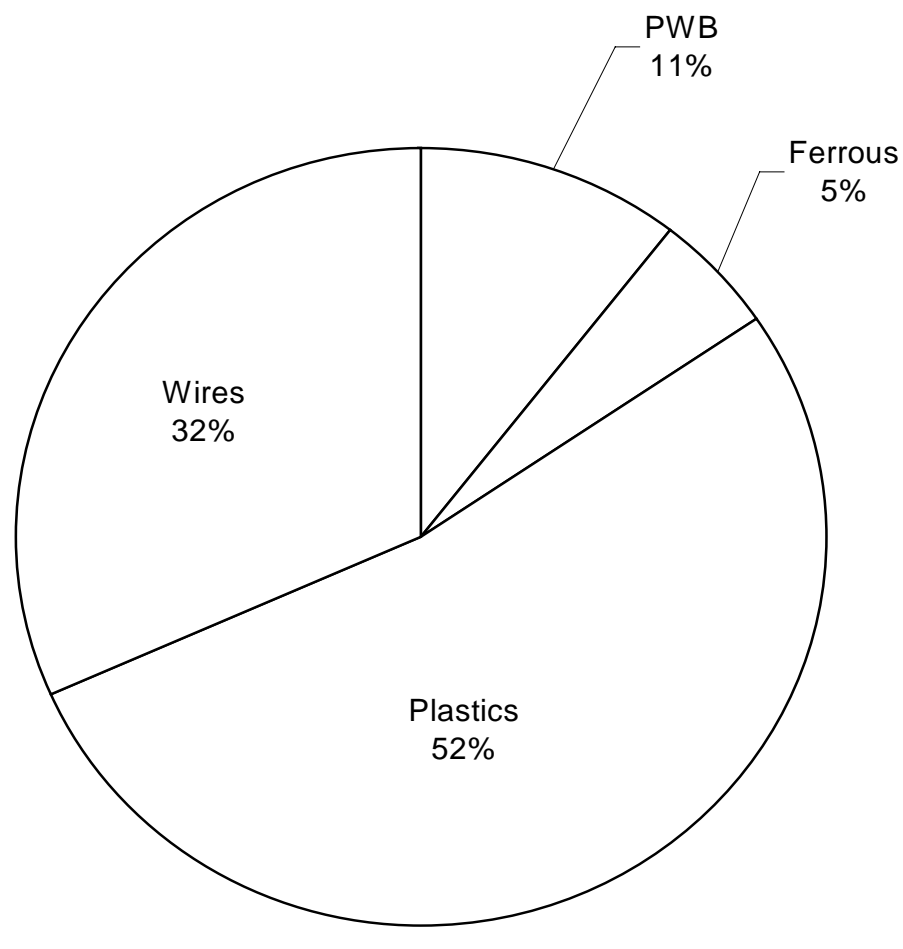

Figure S-9. Average Composition of Mice Tested 


\begin{tabular}{|c|c|c|c|c|c|c|c|}
\hline \multirow[t]{2}{*}{$\begin{array}{l}\text { Sample } \\
\text { Number }\end{array}$} & \multirow[t]{2}{*}{ Make } & \multirow[t]{2}{*}{$\begin{array}{c}\text { Model/ } \\
\text { Year }\end{array}$} & \multirow[t]{2}{*}{ Method } & \multirow[t]{2}{*}{ pH } & \multicolumn{3}{|c|}{$\begin{array}{l}\text { Metal Concentration } \\
\text { in Leachate }(\mathrm{mg} / \mathrm{L})\end{array}$} \\
\hline & & & & & $\mathbf{F e}$ & $\mathbf{P b}$ & $\mathbf{Z n}$ \\
\hline 1 & Dexxa & 9MD/1987 & (True) & 5.0 & 9.11 & 10.0 & 16.3 \\
\hline 2 & Logitech & $\begin{array}{c}\text { 3F-HH/ } \\
1987\end{array}$ & (True) & 5.0 & 75.0 & 38.1 & 16.8 \\
\hline 3 & Logitech & M30 9F & (True) & 5.0 & 45.5 & 16.2 & 10.9 \\
\hline 4 & Manhatan & N/A & (True) & 4.9 & 9.45 & 7.90 & 0.282 \\
\hline 5 & Microsoft & $58264 / 1994$ & (True) & 5.0 & 26.9 & 33.4 & 0.248 \\
\hline 6 & Logitech & M-C43 & (True) & 5.0 & 11.2 & 41.2 & 4.61 \\
\hline 7 & Logitech & M30 9F & (True) & 5.0 & 15.1 & 6.39 & 0.145 \\
\hline 8 & Microsoft & $58266 / 1994$ & (True) & 5.0 & 50.6 & 13.1 & 0.339 \\
\hline 9 & Microsoft & 92841 & (True) & 5.0 & 55.3 & 12.1 & 0.157 \\
\hline 10 & Microsoft & $58269 / 1994$ & (True) & 5.1 & 7.39 & 30.5 & 0.388 \\
\hline 11 & Microsoft & $58269 / 1994$ & (True) & 5.0 & 23.4 & 6.87 & 24.4 \\
\hline 12 & Microsoft & $58269 / 1994$ & (True) & 5.0 & 65.7 & 28.0 & 7.46 \\
\hline 13 & Microsoft & $52695 / 1994$ & (True) & 5.0 & 4.95 & 6.73 & 3.89 \\
\hline 14 & Microsoft & $52695 / 1994$ & (True) & 5.0 & 19.3 & 15.2 & 9.73 \\
\hline 15 & Microsoft & $58269 / 1994$ & (True) & 5.0 & 17.5 & 21.8 & 0.239 \\
\hline
\end{tabular}




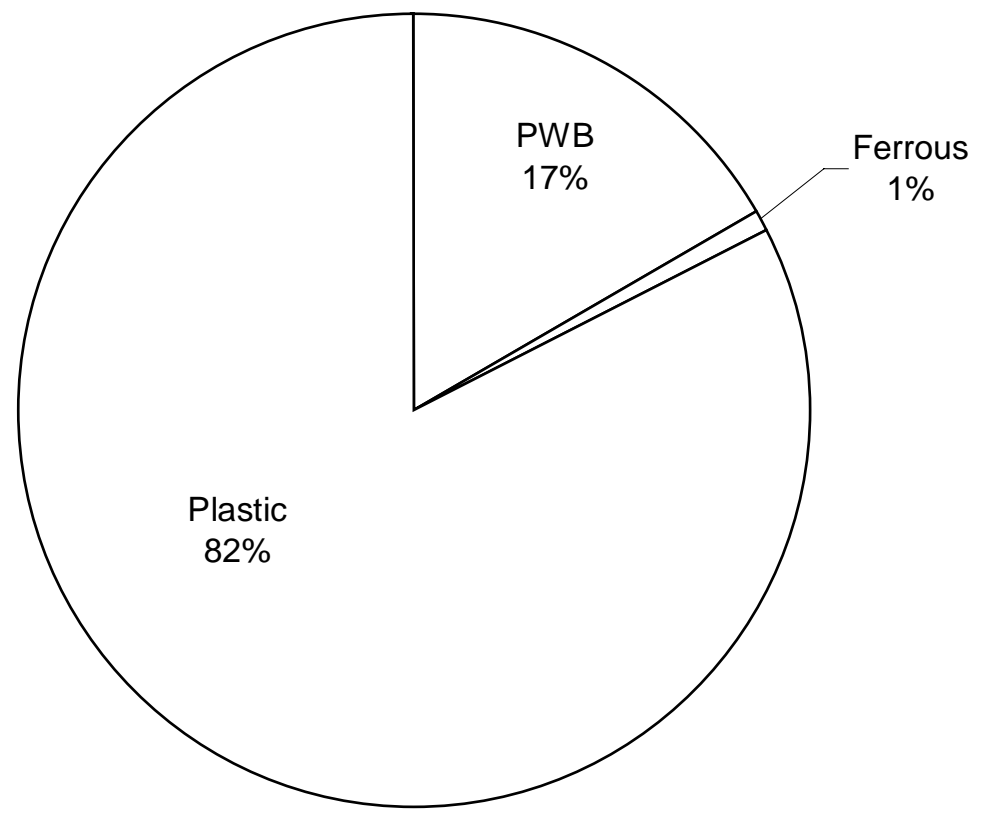

Figure S-10. Average Composition of Remote Controls Tested 


\section{Table S-10. TCLP Results for Remote Controls}

\begin{tabular}{cccccccc}
$\begin{array}{c}\text { Sample } \\
\text { Number }\end{array}$ & Make & Model/Year & Method & pH & \multicolumn{3}{c}{$\begin{array}{c}\text { Metal Concentration in } \\
\text { Leachate }(\mathbf{m g} / \mathbf{L})\end{array}$} \\
& & & & & $\mathbf{F e}$ & $\mathbf{P b}$ & $\mathbf{Z n}$ \\
1 & Memorex & 4900 & True & 5.1 & 67.3 & 11.4 & 0.200 \\
2 & JVC & RM-RX130 & True & 5.0 & 12.5 & 10.0 & 3.50 \\
3 & SHARP & G0956GE & True & 5.0 & 16.7 & 14.0 & 1.70 \\
4 & RCA & $949 T$ & True & 5.0 & 20.0 & 33.3 & 2.18 \\
5 & Hitachi & Vt-RN361A & (M.S.) & 5.0 & 13.8 & 5.20 & 1.50 \\
6 & JVC & RM-C688 & (M.S.) & 4.9 & 3.241 & 7.30 & 0.160 \\
7 & JVC & RM-C689 & (M.S.) & 5.0 & 18.5 & 6.00 & 3.32 \\
8 & Magnavox & NE001UP & (M.S.) & 4.9 & 23.9 & 5.70 & 3.30 \\
9 & RCA & N/A & (M.S.) & 5.0 & 0.850 & 7.60 & 0.130 \\
10 & Hitachi & CLU-241 & (M.S.) & 5.0 & 14.0 & 20.0 & 3.00 \\
11 & UEI & 3021W & True & 5.0 & 24.5 & 1.35 & 0.550
\end{tabular}




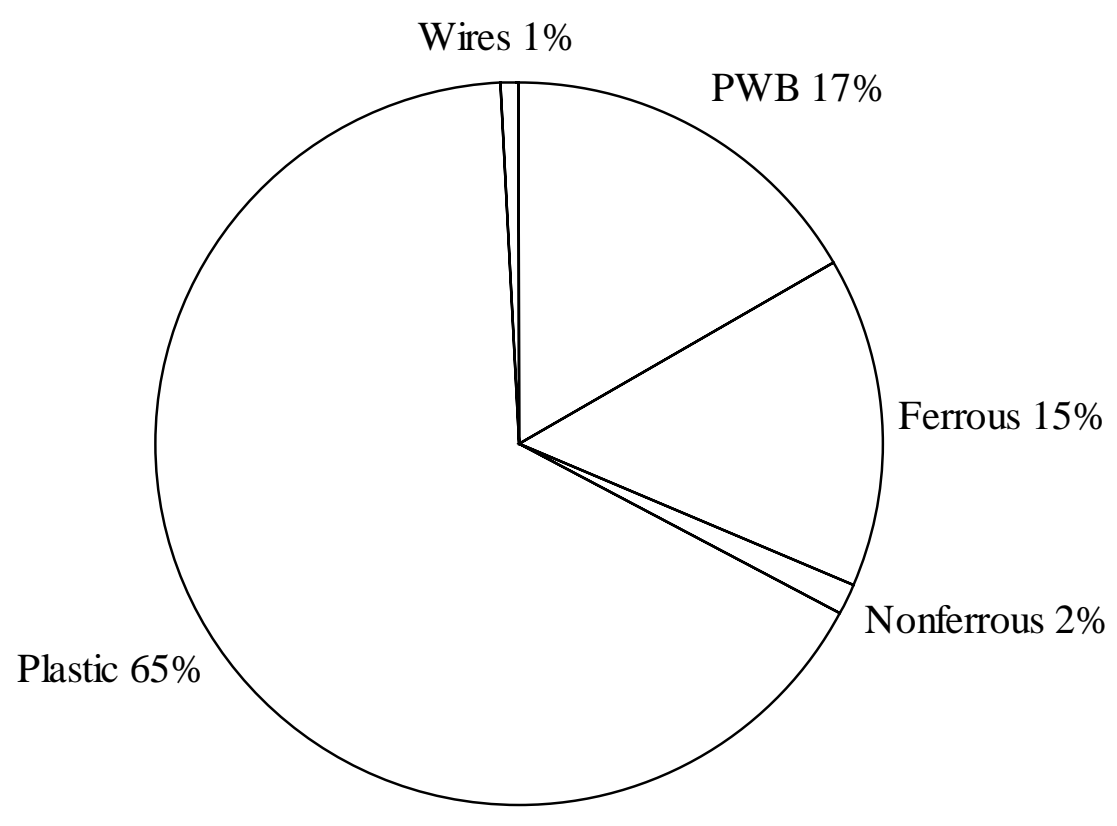

Figure S-11. Average Composition of Smoke Detectors Tested 


\begin{tabular}{|c|c|c|c|c|c|c|c|}
\hline \multirow{3}{*}{$\begin{array}{l}\text { Sample } \\
\text { Number }\end{array}$} & \multicolumn{5}{|c|}{ Table S-11. TCLP Results for Smoke Detector } & & \\
\hline & \multirow[t]{2}{*}{ Make } & \multirow[t]{2}{*}{ Model } & \multirow[t]{2}{*}{ Method } & \multirow[t]{2}{*}{ pH } & \multicolumn{3}{|c|}{$\begin{array}{l}\text { Metal Concentration in } \\
\text { Leachate }(\mathrm{mg} / \mathrm{L})\end{array}$} \\
\hline & & & & & $\mathbf{F e}$ & $\mathbf{P b}$ & $\mathbf{Z n}$ \\
\hline 1 & First Alert & SA301 & True & 5.0 & 28.0 & 44.0 & 1.02 \\
\hline 2 & Square D & SD631 & True & 5.0 & 63.5 & 51.1 & 8.04 \\
\hline 3 & Fire $\mathrm{X}$ & X6-6 & True & 5.0 & 5.49 & 38.3 & 7.20 \\
\hline 4 & Square D & 4919 & True & 5.0 & 42.2 & 13.7 & 10.1 \\
\hline 5 & Dicon Systems & $330 \mathrm{~L}$ & True & 5.1 & 26.3 & 16.9 & 2.37 \\
\hline 6 & Fire $\mathrm{X}$ & G-6 & True & 5.2 & 66.9 & 17.4 & 0.670 \\
\hline 7 & Fire $X$ & FX1020 & True & 5.2 & 4.94 & 1.09 & 190 \\
\hline 8 & Square D & EDG-4S & True & 5.1 & 35.0 & 0.117 & 78.8 \\
\hline 9 & Honeywell & TC805C1000 & True & 4.9 & 0.809 & 24.1 & 0.230 \\
\hline 10 & Walter-Kidde & K-202 & True & 5.0 & 36.0 & 0.237 & 183 \\
\hline
\end{tabular}




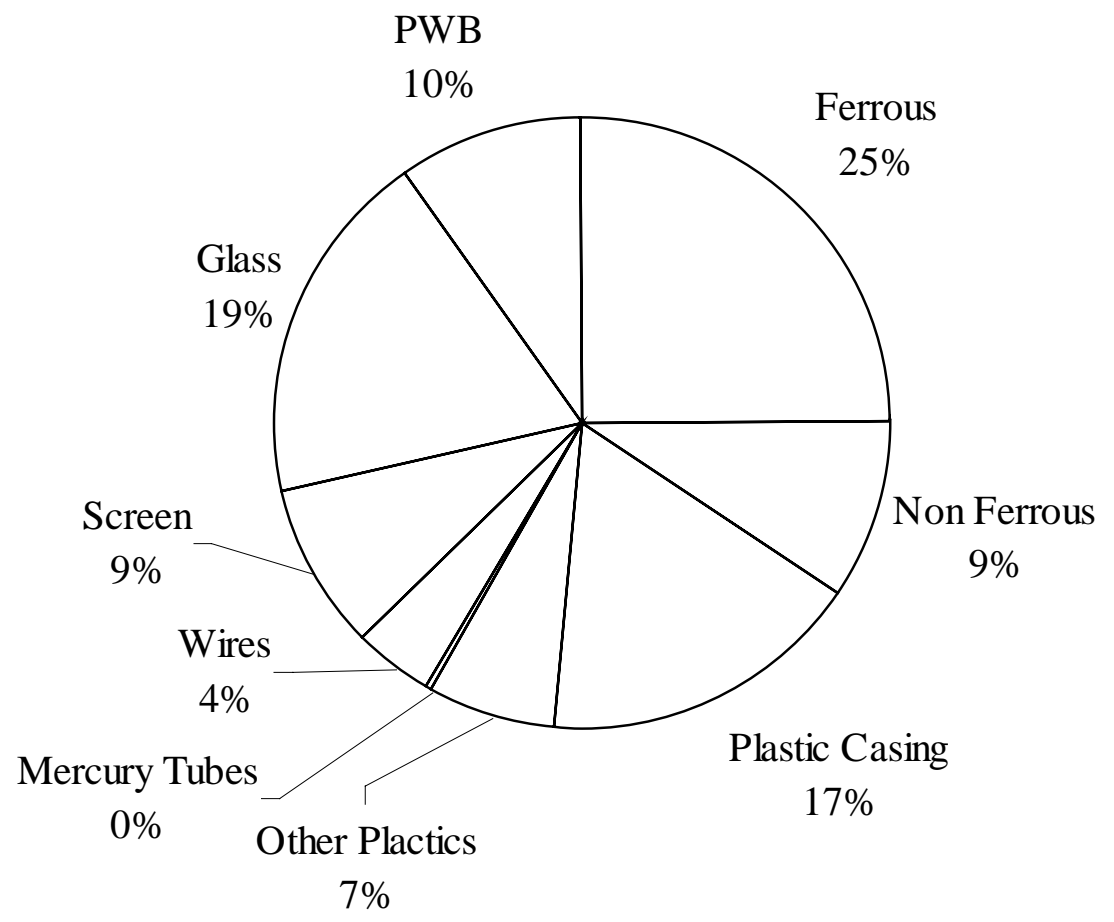

Figure S-12. Average Composition of Flat Panel Displays 


\begin{tabular}{|c|c|c|c|c|c|c|c|}
\hline \multirow[t]{2}{*}{$\begin{array}{l}\text { Sample } \\
\text { Number }\end{array}$} & \multirow[t]{2}{*}{ Make } & \multirow[t]{2}{*}{ Model } & \multirow[t]{2}{*}{ Method } & \multirow[t]{2}{*}{$\mathbf{p H}$} & \multicolumn{3}{|c|}{$\begin{array}{l}\text { Metal Concentration in } \\
\text { Leachate }(\mathrm{mg} / \mathrm{L})\end{array}$} \\
\hline & & & & & $\mathbf{F e}$ & $\mathbf{P b}$ & $\mathbf{Z n}$ \\
\hline 2 & Dell Flatop & 1703FP & (L) & 5.20 & 183 & 3.08 & 72.4 \\
\hline 3 & Apple Studio & M4551 & (L) & 5.02 & 37.1 & 0.324 & 85.2 \\
\hline 4 & Dell Flatop & $1703 \mathrm{FP}$ & (L) & 5.19 & 288 & 6.43 & 114 \\
\hline 5 & Dell Flatop & $1703 \mathrm{FP}$ & (L) & 5.16 & 267 & 6.00 & 117 \\
\hline 6 & IBM & 9513-AW1 & (L) & 5.02 & 36.6 & 1.18 & 92.5 \\
\hline 7 & Dell Flatop & $1703 \mathrm{FP}$ & (L) & 5.23 & 360 & 4.54 & 113 \\
\hline 8 & Dell Flatop & $1703 \mathrm{FP}$ & (L) & 5.20 & 274 & 4.90 & 62.1 \\
\hline 9 & Dell Flatop & $1703 \mathrm{FP}$ & (L) & 5.03 & 126 & 3.60 & 105 \\
\hline 10 & IBM & 24U FK01 & (L) & 4.96 & 167 & 2.71 & 67.4 \\
\hline
\end{tabular}

Note: Sample 1 not reported due to loss from test vessel leakage. 


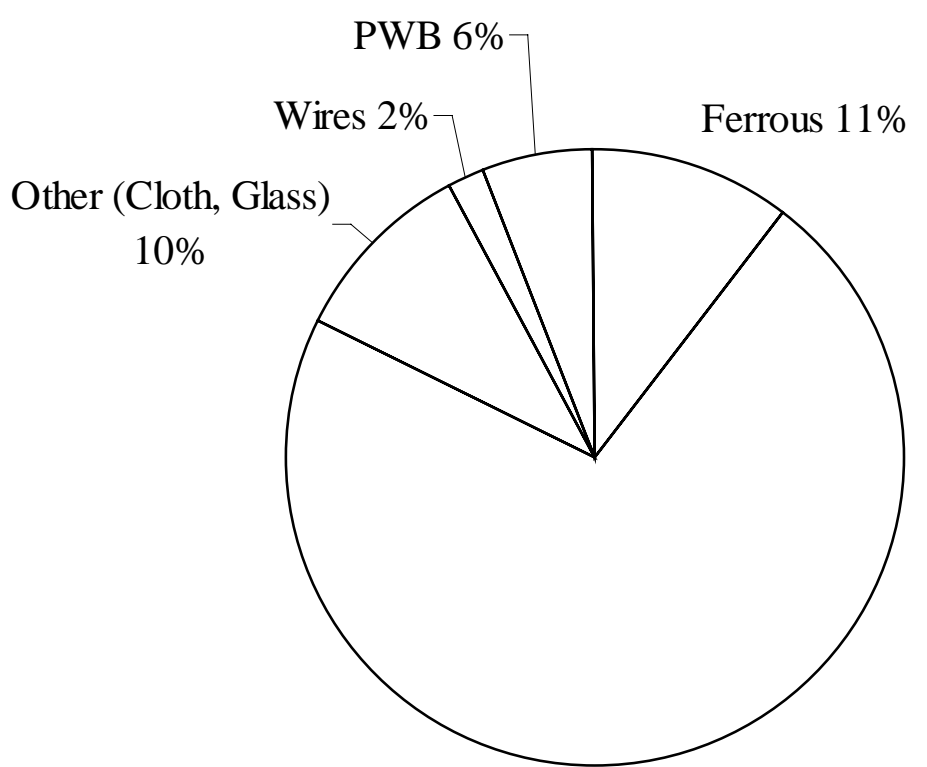

Plastic $71 \%$

Figure S-13. Average Composition of Electronic Toys 


\section{Sample \\ Number}

$\begin{array}{ll}1 & \\ 2 & \\ 3 & \text { Fisher Price } \\ 4 & \text { Fisher Price } \\ 5 & \text { Vtech }\end{array}$

$6 \quad$ Fisher Price

7 Radica

8 Deluxe Sport

Games

9 Deluxe Sport Games

$10 \quad$ Kid

Connection

Table S-13. TCLP Results for Electronic Toys Model Method pH Metal Concentration in Leachate $(\mathrm{mg} / \mathrm{L})$

$\begin{array}{cccccc} & & & \mathbf{F e} & \mathbf{P b} & \mathbf{Z n} \\ \text { Teddy Bear } & \text { True } & 5.1 & 44.5 & 1.42 & 81.9 \\ \text { Groove } & \text { True } & 5.1 & 35.0 & 0.684 & 137 \\ \text { Connection } & & & & & \\ \text { ABC Bus } & \text { True } & 5.0 & 111 & 7.61 & 2.38 \\ \text { Phone } & \text { True } & 5.0 & 63.2 & 29.4 & 6.98 \\ \text { Sqwalky } & \text { True } & 5.1 & 81.5 & 20.5 & 17.8 \\ \text { Talky } & & & & & \\ \text { Happy Face } & \text { True } & 5.0 & 37.9 & 11.5 & 2.12 \\ \text { Slot Pocket } & \text { True } & 5.0 & 161 & 29.5 & 13.5 \\ \text { Soccer } & \text { True } & 5.0 & 75.0 & 17.0 & 8.19 \\ \text { Football } & \text { True } & 5.1 & 67.4 & 29.8 & 2.91 \\ \text { Cash } & \text { True } & 5.0 & 68.9 & 7.98 & 0.214 \\ \text { Register } & & & & & \end{array}$

